\title{
Bi-layered nano active implant with hybrid stem cell microtissues for tuned cartilage hypertrophy
}

\begin{abstract}
Repair of articular cartilage defects remains challenging, as a majority of these defects reach the subchondral bone. The objectives of this work were first, to develop a new advanced therapeutic implant for osteoarticular repair, and second, to prevent hypertrophy of the articular cartilage region. For this aim, we developed a bicompartmented implant, presenting a first layer for subchondral bone regeneration: Compartment 1, composed of a nanofibrouspoly ( $\varepsilon$-caprolactone) membrane equipped with BMP-7 growth factor nanoreservoirs, second layer for cartilage regeneration: Compartment 2, composed of alginate/hyaluronic acid hydrogel. To modulate hypertrophy, compartment 2 was seeded with microtissues of human mesenchymal stem cells co-cultured with human chondrocytes as differentiation inductors. This new strategy offers an appropriate double 3D environment (microtissues in Hydrogel) as inductive trigger for cartilage regeneration with tuned hypertrophy.
\end{abstract}

Keywords: regenerative nanomedicine, nanofibrous pcl implant, human mesenchymal stem cells (hmscs); microtissues (mts), hypertrophy, bmp-7
Volume I Issue I - 2015

\author{
Laetitia Keller, ${ }^{1,2}$ Quentin Wagner, ${ }^{1,2}$ Marion \\ Pugliano,' Pierre Breda,' Matthieu Ehlinger,' \\ Pascale Schwinte,' Nadia Benkirane-Jessel ${ }^{1,2}$ \\ 'Osteoarticular and Dental Regenerative Nanomedicine, Faculte \\ de Medecine, France \\ ${ }^{2}$ Universite de Strasbourg, Faculté de Chirurgie Dentaire, France \\ \#These authors have equally contributed to this work, France
}

\begin{abstract}
Correspondence: Nadia Benkirane-Jessel, INSERM UMRI I09, Osteoarticular and Dental Regenerative Nanomedicine, Faculte de Medecine, FMTS, F-67085 Strasbourg, France, Universite de Strasbourg, Faculte de Chirurgie Dentaire, I place de l'Hopital, F-67000 Strasbourg, France, Tel +33 36885337 6, Email nadia.jessel@inserm.fr
\end{abstract}

Received: April 24, 2015 | Published: July 23, 2015
Abbreviations: BMP, bone morphogenic protein; Bsp-II, bone sialoprotein-ii, CHI, chitosan; ECM, extracellular matrix; F, functionalized; FDA, food and drug administration; GAGs, glycosaminoglycans; HA, hyaluronic acid; hCHs, human chondrocytes; hMSCS, human mesenchymal stem cells; LbL, layer by layer; MTs, microtissues; $\mathrm{NF}$, no functionalized; Ocn, osteocalcin; PEG, polyethylene glycol; PGA, poly glycolic acid; PLA, poly lactic acid; PCL, poly $(\varepsilon-$ caprolactone); Runx2, runt-related transcription factor 2; TGF, transforming growth factor

\section{Introduction}

The articular cartilage is a very specific conjunctive tissue divided in several layers, from the articular zone on the articular surface to the mineralized subchondral bone in contact with the surrounding bone. ${ }^{1-3}$ To resist to the compression and provide a low-friction surface for the joint, the osteochondral unit presents a high specific extracellular matrix (ECM) organization in each layer, from high density GAGs (glycosaminoglycanes) absorbing water in the articular zone to a more collagen type I matrix in the subchondral bone..$^{1-3}$ Due to the lack of vascularisation and cells, articular cartilage has a weak capacity for self-repair. Therefore, repair of osteochondral lesions still remains challenging, as the articular cartilage lesion also affects the subchondral bone underneath, and often results in fibrous and frangible repaired tissue. ${ }^{4-8}$ In order to increase the efficiency of articular cartilage repair, it is essential to first repair a solid subchondral bone, supporting articular cartilage regeneration on its surface.

Nanomaterials have a great potential in the field of regenerative medicine, especially in osteoarticular applications. ${ }^{1,9}$ For bone and cartilage, lots of materials have been developed to mimic the physiological extracellular matrix of tissues. They originate from natural substrates (collagen, alginate, gelatin, agarose, fibrin) or are synthetic materials (PEG (polyethylene glycol), PLA (poly lactic acid), PGA (poly glycolic acid). ${ }^{10,11}$ Recently, focus has been made on new generations of nanotechnology-based biomaterials.

As guidance offered by such materials allows the restoration of tissues by mimicking the ECM, nanofibrous scaffolds play a central role in modern strategies in nanomedicine. ${ }^{12,13}$ Our group has developed biodegradable electrospunnanofibrous materials using some FDA (Food and Drug Administration) approved polymer such as poly ( $\varepsilon$-caprolactone) (PCL). ${ }^{14,15}$ Furthermore, the functionalization of these new generation biomaterials, by incorporation of bioactive molecules, increases their efficiency to induce cell differentiation and tissue repair. ${ }^{14-32}$ To this end, we have recently developed new strategies for active therapeutic implants associating FDA approved components. ${ }^{14,15,33-36}$ We have reported the incorporation of active growth factors (TGF $\beta$, BMP-2, BMP-7) as a coating for nanofibrous scaffolds (synthetic or natural). The mechanism of the nanoreservoirs release of active therapeutics is cell contact dependent. These systems are already validated both in vitro and in vivo for the increase of the speed of bone regeneration. ${ }^{14,15,33-37}$

In recent years, in the field of bone and osteoarticular regenerative medicine, the use of adult mesenchymal stem cells (MSCs) provides an increasing interest. Derived from adult tissues, MSCs are defined as self-renewable, multipotent and immunosuppressive. Able to differentiate in chondrocytes or osteoblasts under specific conditions, ${ }^{38-41}$ these stem cells represent ideal candidates for the osteochondral regeneration. Indeed, with autologous chondrocytes, the procurement of a sufficient number of autologous cells is limited and these cells easily lose their phenotype in vitro. ${ }^{8,42,43}$ In recent years, it has been shown that MSCs cultured in a 3D structure, named "micromass" or "pellet", have a more important capacity to differentiate in the chondrogenic way, compared to their use as single cells. ${ }^{44,45}$ Moreover, the use of spheroids (self-assembling microtissues) is known to mimic the embryonic condensation occurring during the 
embryonic endochondral development. ${ }^{46-49}$ Indeed, in recent studies, we have shown that hMSCs, used as single cells but also in 3D wellorganized microtissues (MTs), were able to produce bone in vitro and in vivo when associated with a nanofibrous membrane equipped with BMP-7 nanoreservoirs. ${ }^{35,36}$

In the clinic today, repair strategies (microfracture, mosaicplasty etc...) mostly lead to the formation of fibrocartilage and have limited success, focusing on long-term repair limited to small lesions, and can reveal subchondral bone abnormality. ${ }^{4-8}$ To overcome these clinical drawbacks, osteochondral tissue engineering approaches using multilayered materials for the bone-cartilage unit regeneration (subchondral bone together with articular cartilage) have recently emerged. ${ }^{7,50-55}$ In this work, we focused on subchondral bone and cartilage regeneration and developed an implant composed of two compartments: one for subchondral bone, and the other for articular cartilage regeneration.

To mimic the physiological cues of the bone-cartilage unit, we have used

(i) a nanofibrous polymeric membrane equipped with nanoreservoirs of BMP-7 for the subchondral bone regeneration (Compartment 1)

(ii) Hyaluronic acid/alginate hydrogel for the articular cartilage repair (Compartment 2) seeded with hybrid microtissues (human MSCs combined with human chondrocytes), mimicking the endochondral differentiation development. With the compartment 1 , we achieved the subchondral bone regeneration. We have confirmed that the presence of BMP-7 nanoreservoirs in this compartment (PCL membrane) is a good tool for subchondral bone repair. With the compartment 2 , we achieved cartilage repair with less hypertrophy of the engineered cartilage.

\section{Material and methods}

\section{Bioactive PCL nanofibrous membrane design}

PCL nanofibrous membranes $(50 \mu \mathrm{m}$ thick) were obtained by the electrospinning method as described previously. ${ }^{14,15}$ Addition of BMP7 nanoreservoirs (CHI/BMP-7) ${ }_{6}$ on the nanofibers was performed by the Layer-by-Layer (LbL) technology. ${ }^{37}$ For that, the PCL membrane was dipped successively (6 times during $15 \mathrm{~min}$ ) in alternate solutions of Chitosan (CHI) (Protasan UP CL 113; Novamatrix, Sandvika, Norway; 500mg.mL $\mathrm{mL}^{-1}$ ) and BMP-7 (Euromedex, Souffelweyersheim, France; 200ng. $\left.\mathrm{mL}^{-1}\right)$ prepared in MES buffer (2-N-morpholino ethane sulfonic acid; Sigma-Aldrich Co., St Louis, MO, USA; 0.04M, NaCl $0.15 \mathrm{M})$ at $\mathrm{pH} 5.5$. Each bath was followed by a rinsing step in a solution of MES buffer $(0.04 \mathrm{M}, \mathrm{NaCl} 0.15 \mathrm{M})$.

\section{Cell culture}

Human mesenchymal stem cells (hMSCs) and human chondrocytes (hCHs) (PromoCell, Heidelberg, Germany) were cultured in a proliferation medium (Promocell, Heidelberg, Germany) complemented with supplement mix serum (Promocell, Heidelberg, Germany), 50U. $\mathrm{mL}^{-1}$ of penicillin and $50 \mu \mathrm{g} \cdot \mathrm{mL}^{-1}$ of streptomycin. The cells were incubated at $37^{\circ} \mathrm{C}$ in a humidified atmosphere of $5 \%$ $\mathrm{CO}_{2}$. When cells reached sub-confluence, they were harvested with trypsin and sub-cultured to form microtissues (MTs). To form hMSCs MTs, $3000 \mathrm{hMSCs}$ were cultured in each droplet in suspension of $40 \mu \mathrm{L}$ of medium in 3D culture plate system (GravityPLUS ${ }^{\mathrm{TM}} 3 \mathrm{D}$ Culture, InSphero AG, Zurich, Switzerland) during 5 days. To form hybrid MTs, $1000 \mathrm{hCHs}$ and 2000hMSCs were cultured in a droplet. The same number of formed MTs was then seeded in each nanofibrous PCL membrane (24MTs. $\left.\mathrm{cm}^{-2}\right)$ or in alginate/HA hydrogel.

\section{Culture in alginate/HA hydrogel}

Microtissues (MTs) were suspended in a solution of alginate (Sigma Aldrich) (12mg.mL $\mathrm{mL}^{-1}$ ) and hyaluronic acid (HA) (Lifecore Biomedical, Chaska, USA) $\left(3 \mathrm{mg} \cdot \mathrm{mL}^{-1}\right)$. The alginate/HA solution was then polymerized with a solution of $\mathrm{CaCl}_{2}$ (Sigma Aldrich) at $102 \mathrm{mM}$, during $15 \mathrm{~min}$ at $37^{\circ} \mathrm{C}$. The microtissues embedded in alginate/ HA were then cultured in medium complemented with $1 \mathrm{mM}$ of $\mathrm{CaCl}_{2}$

\section{Microtissues differentiation}

After seeding on PCL nanofibrous membrane functionalized (F) or not (NF) with BMP-7 nanoreservoirs, hMSCs MTs were cultured during 28 days in osteogenic medium consisting in Alpha-MEM complemented with L-glutamine $(2 \mathrm{mM})$, SVF $(10 \%)$, fungizone $\left(250 \mathrm{U} \cdot \mathrm{mL}^{-1}\right)$, penicilline-streptomycine $\left(10 \mu \cdot \mathrm{mL}^{-1}\right)$, ascorbic acid $(60 \mu \mathrm{m}), \beta$-glycerophosphate $(10 \mathrm{mM})$ and dexamethasone $(10 \mathrm{nM})$. After seeding in alginate/HA hydrogel, hybrid MTs were cultured during 28 days in hMSCs proliferation medium (Promocell, Heidelberg, Germany), and hMSCs MTs were cultured during 28 days in chondrogenic medium consisting in Alpha-MEM complemented with L-glutamine $(2 \mathrm{mM})$, SVF $(10 \%)$, fungizone $\left(250 \mathrm{U} \cdot \mathrm{mL}^{-1}\right)$, penicilline-streptomycine $\left(10 \mu . \mathrm{mL}^{-1}\right)$, L-Proline $\left(40 \mu \mathrm{g} . \mathrm{mL}^{-1}\right)$, dexamethasone $(10 \mathrm{nM})$ and ascorbic acid $\left(50 \mu \mathrm{g} \cdot \mathrm{mL}^{-1}\right)$.

\section{Indirect immunofluorescence}

Samples were fixed with $4 \%$ paraformaldehyde (PFA) and were then treated with a solution of $0.1 \%$ Triton and $1 \%$ BSA for saturation and permeabilisation. Primary antibodies were then added during 2 hours at room temperature at the $1 / 200$ concentration. Primary antibodies used were: rabbit anti-Runx2 (Sigma Aldrich), mouse antiOsteocalcin (Ocn) (Santa Cruz Biotechnology, Dallas, TX, USA), and rabbit anti-BspII Sigma Aldrich). After rinses with PBS, 488 Alexa Fluor anti-rabbit or anti-mouse (Molecular Probes; Life Technologies, Fisher Scientific, Illkirch, France) (1/200) were added during 1 hour at room temperature. PBS rinses were made and phalloidin (Molecular Probes; Life Technologies) (1/200) was added during 20min at room temperature. A DAPI (Sigma-Aldrich) solution $(200 \mathrm{nM})$ was then added to the sample to observe cell nuclei. After mounting with Dako $^{\circledR}$ (Dako, Courtaboeuf, France), the samples were observed with an epifluorescent microscope (Leica DM 4000B).

\section{Alamar Blue $\AA^{\circledR}$ test}

To analyse the metabolic activity of hMSCs MTs, the Alamar Blue $^{\circledR}$ test (Thermo Fisher Scientific, Waltham, MA, USA) was used. For that, the cells were cultivated during 4 hours in a solution of $10 \%(\mathrm{v} / \mathrm{v})$ Alamar Blue ${ }^{\circledR}$ in a complete medium without phenol red. Duplicates of each culture medium well were then analysed with a spectrometer (FC Multiskan) at the 570 and $595 \mathrm{~nm}$ wavelengths. For each test, $\mathrm{n}=3$. The $\mathrm{p}$ value was determined by a $t$-paired test.

\section{Quantitative RT-PCR}

For the MTs grown in the alginate/HA-polymerized hydrogel, samples were first dissolved in a solution of citrate $(55 \mathrm{mM})$, $\mathrm{NaCl}(0,15 \mathrm{M})$ and EDTA $(30 \mathrm{mM})$. The total RNA was extracted from the MTs with the high pure RNA Isolation Kit (Roche). The RetroTRanscription was performed with the iScript ${ }^{\mathrm{TM}}$ reverse 
Transcription Supermix (Bio-Rad, Marnes-la-Coquette, France). The Real-time PCR reaction were then carried out using the $\mathrm{iTaq}{ }^{\mathrm{TM}}$ Universal SYBR ${ }^{\circledR}$ green super mix (Bio-Rad) and the CFX cycler system (Bio-Rad) with the following cycle condition: an initial denaturation step of $95^{\circ} \mathrm{C}$ for 2 min was performed, followed by 39 cycles of denaturation at $95^{\circ} \mathrm{C}$ for 5 seconds, annealing at $60^{\circ} \mathrm{C}$ during 30 seconds and extension at $65^{\circ} \mathrm{C}$ for 5 seconds. For each test, $\mathrm{n}=3$. Statistical significance was determined by a $t$ paired test (Table 1 ).

Table I The primers used

\begin{tabular}{lll}
\hline Target gene & Forward & Reverse \\
\hline ACTIN B & 5'-GATGAGATTGGCATGGCTTT-3' & 5'-CACCTTCACCGTTCCAGTTT-3' \\
RUNX2 & 5'-CCAACCCACGAATGCACTATC-3' & 5'-TAGTGAGTGGTGGCGGACATAC-3' \\
\hline
\end{tabular}

\section{In vivo experimentation}

Animals and tissues: All procedures were designed in compliance with the recommendations of the European Union (2010/63/EU) for the care and use of laboratory animals. Ethics statement: Experiments followed current European Union regulations (Directive 2010/63/ EU), and were performed according to authorized investigator Dr. N. Jessel (Director of the "Osteoarticular and Dental Regenerative Nanomedicine" Team), holder of a personal license from "Prefecture du Bas-Rhin" (No. 67-315), who oversaw experiments done on mice. All experiments were done in the "Animalerie Centrale de la Faculte de Medecine de Strasbourg" with the approval number: A 67-482-35 from the Veterinary Public Health Service of the "Prefecture du BasRhin", representing the French Ministry of Agriculture, Department of Veterinary Science. For further tissues implantations, all surgery was performed under Ketamine and Xylazine anesthesia, and all efforts were made to minimize suffering. Nude male mice (CTR: NIHFoxn1nu, Charles River, France), 6 weeks of age, were anesthetized with intra-peritoneal injection of $100 \mathrm{mg}^{\mathrm{kg}} \mathrm{kg}^{-1}$ of ketamine (VIRBAC Sante Animale, Centravet) and $10 \mathrm{mg} \cdot \mathrm{kg}^{-1}$ of Xylazine (Rompun ${ }^{\circledR}$ $2 \%$, Centravet). The nano structured bioactive implant was implanted behind the ears of mice, between skin and muscles. After 90days, the animals were sacrificed with an intra-peritoneal injection of lethal dose of ketamine, to examine the explant by histological staining.

\section{Histological staining of glycosaminoglycanes}

Samples of alginate hydrogel were first fixed with a solution of $4 \%$ para formaldehyde (PFA) containing $100 \mathrm{mM}$ of cacodylatetrihydrate and $10 \mathrm{mM}$ of $\mathrm{CaCl}_{2}$. Samples were then embedded in paraffin for serial sections of $7 \mu \mathrm{m}$. For Safranin $\mathrm{O} /$ Fast green staining, the samples were rinsed with PBS and dipped in a solution of Fast green $(0,02 \% \mathrm{~m} / \mathrm{v})$, acetic acid $(1 \% \mathrm{~m} / \mathrm{v})$ and finally Safranin O $(0,1 \% \mathrm{~m} / \mathrm{v})$. For Alcian Blue staining, the samples were rinsed with distilled water and a solution of $2 \%(\mathrm{~m} / \mathrm{v})$ Alcian Blue (Sigma-Aldrich) at $\mathrm{pH} 4.2$ was added during 2 hours at room temperature. Samples were then rinsed with distilled water, dehydrated and mounted with Histolaque LMR $^{\circledR}$ (Labo Moderne, France) to be observed with bright field microscope (Leica DM 4000B).

\section{Results and discussion}

PCL membrane equipped with BMP-7 nanoreservoirs for subchondral bone repair (compartment I)

In bone regenerative medicine field, bioactive biomaterials mimicking the extracellular matrix have been developed. ${ }^{56,57}$ As examples, Medtronic offers InductOs ${ }^{\circledR}$, which is a basic collagen matrix (of animal origin) soaked in osteogenic growth factor BMP-2 (12mg), and Stryker $^{\circledR}$ offers the same collagen membrane soaked in BMP-7.58,59 Despite their application in clinic, the release of these active molecu- les from these materials is passive and not controlled. Recently, we have developed a nano reservoirs technology, on synthetic or natural membranes ${ }^{14,15,33,35,36}$ with active cell contact dependent release. The advantage of this technology resides in the fact that this material generates an active release of molecules dependent on the cells' adhesion on the nano reservoirs and their cell-dependent degradation, ${ }^{14}$ leading to an increase of growth factor bioavailability after implantation. For bone tissue repair, we have previously shown that BMP-7 nano reservoirs on collagen membrane (Bio Gide ${ }^{\circledR}$ from Geistlich) were able to increase mineralization of human osteoblasts and mesenchymal stem cells (hMSCs), seeded as single cells or as microtissues (MTs) ${ }^{35,36}$ The replacement of this natural material (of animal origin) by a synthetic polymer, can be a key to avoid inflammatory response and rejection in vivo. For that purpose, we developed electro spunnano fibrous membranes based on the poly ( $\varepsilon$-caprolactone) (PCL), which is an FDA approved polymer. ${ }^{14,15}$ This material, when equipped with nano reservoirs of BMP-2, can increase the speed of bone marker expression in vitro after 14 days and in vivo..$^{14,15}$ As a strategy to enhance subchondral bone regeneration efficiency in vivo, we used BMP-7 functionalized (F) nanofibrous PCL membrane and human mesenchymal stem cells. To confirm the osteogenic differentiation of hMSCs MTs on functionalized (F) PCL membrane, these hMSCs MTs were cultured in osteogenic medium during 28 days (Figure 1). The PCL (F) membrane was biocompatible with hMSc MTs, as the cells metabolic activity increased all along the culture (Figure 1A). In the literature, it is reported that BMP-7 acts as an attenuator of cell cycling during early osteogenic differentiation of $\mathrm{hMSCs}^{60}$ and our results are in accordance with previous observations. ${ }^{35,36}$ After 14 days, hss MTs were able to express bone specific markers (Runx2, Ocn, BspII) on the (NF) and (F) PCL membrane (Figure 1B). Runx2, a transcription factor induced when mesenchymal cells are in the bone differentiation way was expressed in the two conditions (F, NF). Interestingly, Runx2 and Osteocalcin (Ocn) seem to be more important in the presence of the nanoreservoirs (NRs) (Figure 1B). These results were in accordance with expected results as we previously showed the osteogenic differentiation of hMSCs as single cells with BMP-7 NRs, ${ }^{36}$ or as MTs. ${ }^{35}$ As collagen represented a good tool for hMSCs MTs differentiation, ${ }^{35}$ the PCL electro spunnano fibrous membrane equipped with BMP-7 nano reservoirs can represent a good support for these cells and for bone regeneration in vivo. With this strategy, we can overcome the current approaches used in the clinic today (Stryker collagen soaked in BMP-7) for bone repair, leading to overdosing due to the massive and passive release of BMP-7, inducing adverse side effects.

\section{Hybrid MTs in alginate/HA hydrogel for cartilage re- generation with tuned hypertrophy (Compartment 2)}

To engineer cartilage on the bone surface functionalized support (compartment 1), we used Alginate/HA hydrogel seeded with stem cells to mimic the articular cartilage compartment. First, hMSCs in 
well-organized MTs were used to mimic the condensation during the endochondral differentiation way during embryonic development. ${ }^{46-49}$ Already used in the cartilage-engineering field, MSCs cultured in a 3D configuration (micromass, pellet) show increased chondrogenic differentiation compared to MSCs single cells..$^{44,45}$ Moreover, it was shown that co-culture of MSCs with chondrocytes induces a chondrogenic differentiation of MSCs with less hypertrophic markers. ${ }^{61-64}$ On the other hand, single cells and 2D culture systems can dedifferentiate chondrocytes via fibroblastic phenotype. ${ }^{65,66}$ Rather than performing culture with growth factors, culture modes with cellular interactions have been shown to generate more physiological cues and doses. The paracrine signal between chondrocytes and MSCs is dependent on the distance between cells and an excessive distance blocks their interaction. ${ }^{67}$

In this work we chose to increase these cell-cell interactions by using a co-culture of two kinds of cells (hMSCs and hCHs) in microtissues (MTs). Having shown that hMSCs MTs in HA/hydrogel can represent a good tool for chondrogenic differentiation, the next step was to analyse the effect of hCHs on hMSCs in MTs (Figure 2 ). For that purpose, hybrid MTs (hMSCs/hCHs) were cultured in alginate/HA hydrogel during 28 days in non chondrogenic medium (MSC proliferation medium). The glycosaminoglycanes (GAGs) expression and the hypertrophic marker Runx2 were then evaluated in the MTs (Figure 2B \& 2C). We first observed the chondrogenic differentiation of these hybrid MTs by staining specific GAGs (Figure 2B). QRT-PCR was then used to compare the expression of
RUNX2 in hybrid MTs and in hMSCs MTs, and revealed a significant decrease in RUNX2 in the MTs containing hCHs (hybrid MTs) (Figure $2 \mathrm{C})$. The use of hCHs in combination with hMSCs in MTs allows inducing a less hypertrophic phenotype. Alginate polymeric material was already shown as beneficial for cartilage engineering and repair, and has been used for the transplantation of cells in several clinical trials. ${ }^{11,68,69}$ Indeed, this unmodified hydrogel, which has attractive properties such as immobilization of cells, can also benefit to the chondrogenic differentiation because it does not promote mammalian cell attachment and proliferation..$^{70,71}$ Using alginate hydrogel to mimic the natural environment of articular cartilage, associated with co-cultured $\mathrm{hMSCs} / \mathrm{hCHs}$ in a tridimensional well organized conformation (hybrid MTs), is a good strategy to regenerate articular cartilage tissue with less hypertrophy.

\section{Nano functionalized compartmented implant design (compartment I+ compartment 2)}

A majority of articular cartilage defects reach the subchondral bone and articular cartilage repair is done on frangible subchondral bone..$^{4-8}$ To increase the efficiency of cartilage repair we have used, not only an alginate hydrogel (compartment 2) seeded with hybrid MTs for cartilage repair, but also a functionalized membrane (compartment 1) for subchondral bone regeneration. By using this innovative strategy, our proposed therapeutic medical device associates two compartments in order to reproduce the bone-cartilage unit (Figure 3A).
A

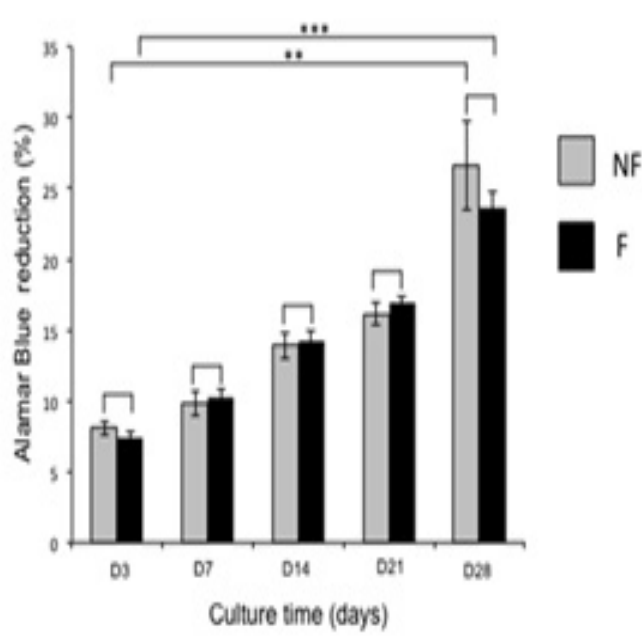

B

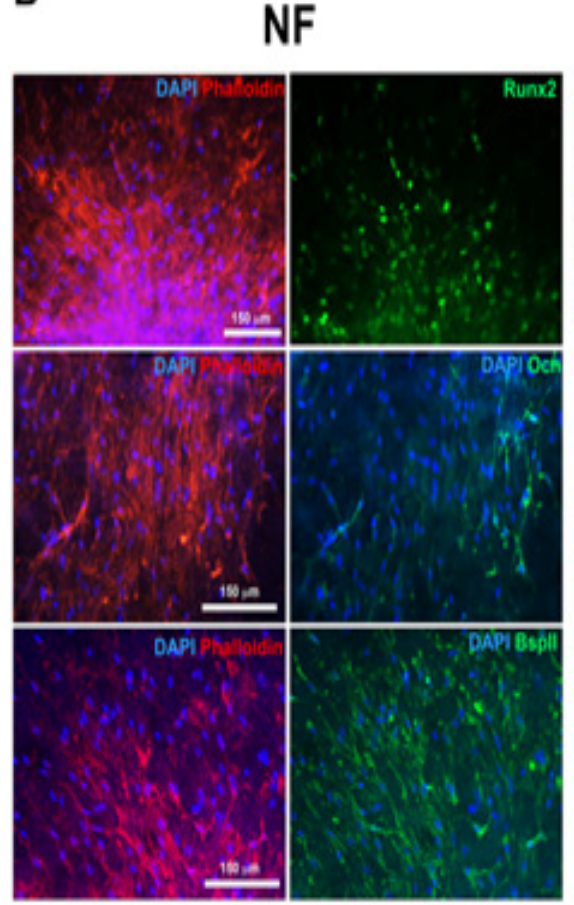

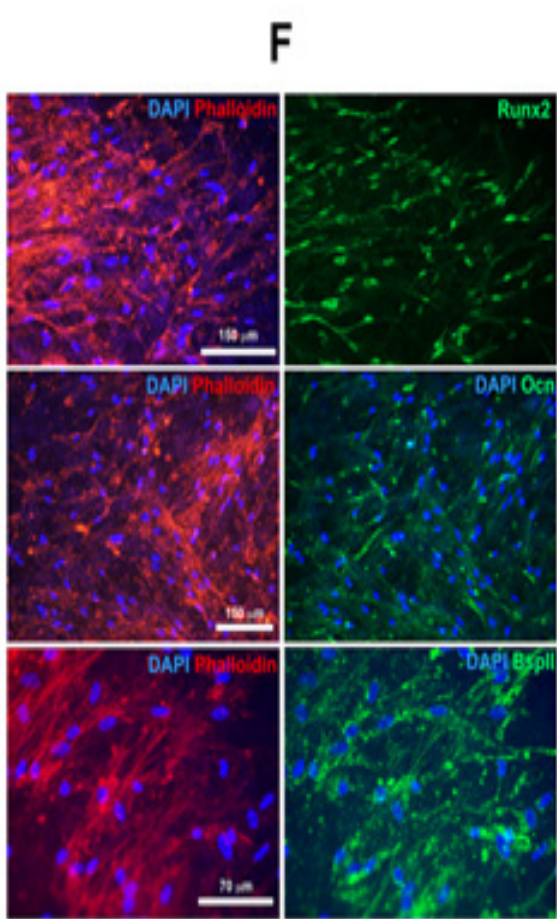

Figure I Osteogenicbehaviour of hMSCs MTs on nanofibrous PCL equipped with (CHI-BMP-7) nanoreservoirs (compartment I). (A) Metabolic activity was measured by an Alamar Blue ${ }^{\circledR}$ test after 3, 7, I4, 2 I and 28 days. $n=3$. All values are expressed as mean $+/$ - SEM. Differences were considered significant at $95 \%$ confidence level $(* * * P<0.0 I=$ extremely significant; $* * P<0.05=$ very significant, $* P<0 . I=$ significant $)$. No significant differences were observed between cells growing on functionalized (F) and not functionalized (NF) nanofibrous membrane (t paired test). (B) Expression of proteins specific to the bone tissue for hMSCs MTs growing on functionalized (F) and not functionalized (NF) nanofibrous PCL membrane after 14days in vitro. Runx2, Ocn (Osteocalcin) and Bspll (Bone sialoprotein II) were visible in green after indirect immunofluorescence. The nuclei were stained in blue with DAPI and actin in red with phalloidin. 


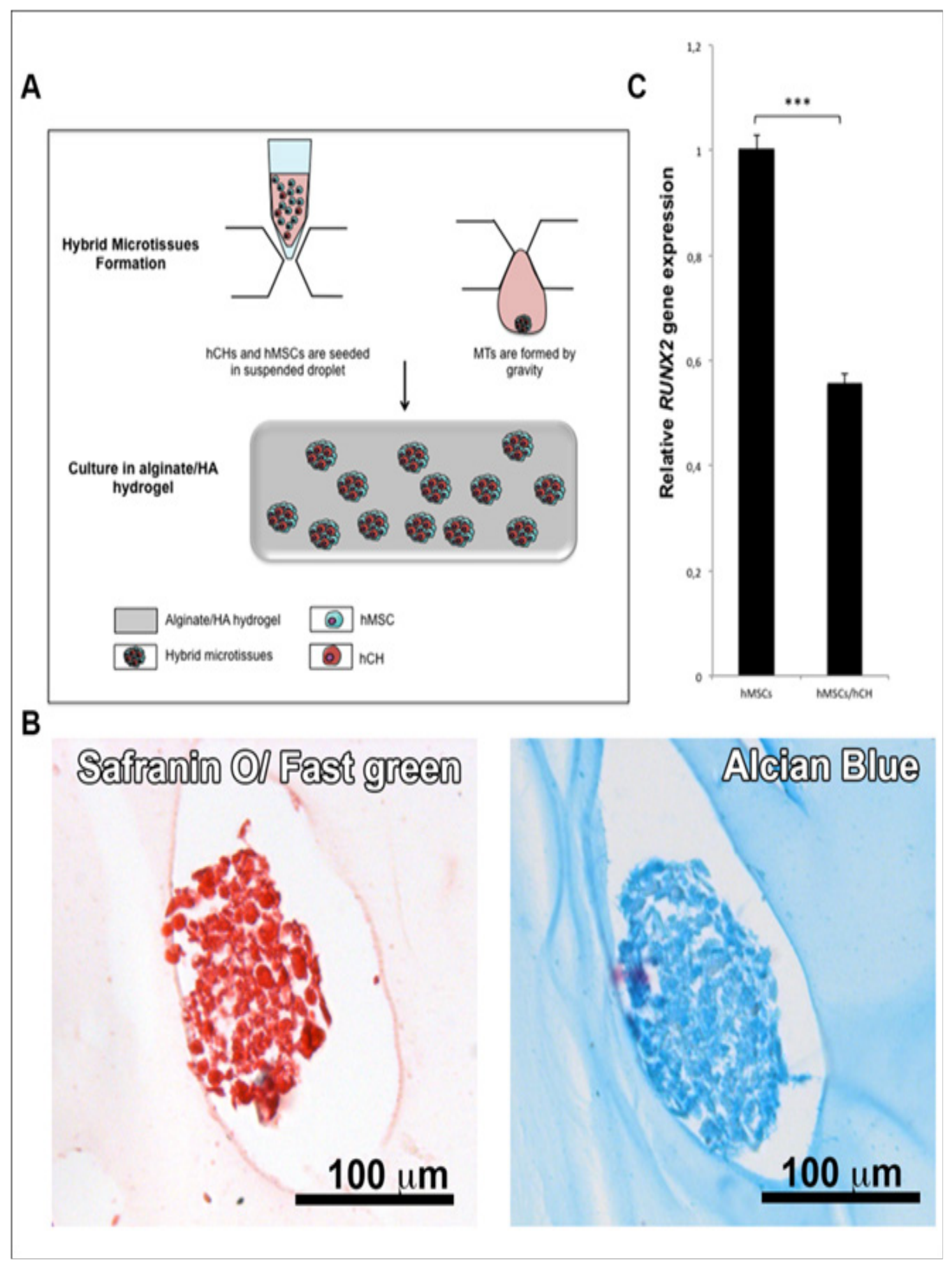

Figure 2 Chondrogenic behaviour and hypertrophy evaluation of hybrid MTs in alginate/HA hydrogel (compartment 2). (A) Hybrid MTs were formedby culturing $\mathrm{hCHs}$ and $\mathrm{hMSCs}$ in droplet suspension in a none chondrogenic medium. After 5 days, hybrid MTs were embedded in alginate/Ha hydrogel during 28days without chondrogenic medium. (B) Histological staining of hybrid MTs specific to glycosaminoglycans visible in blue (Alcian Blue) and in red (Safranin 0/ Fast Green). (C) Relative expression of the hypertrophic marker RUNX2 for hybrid MTs compared to hMSCs MTs in chondrogenic medium after 28 days. RUNX2 is significantly decreased in the hybrid MTs compared to the hMSCsMTs. $n=3$. All values are expressed as mean $+/$ - SEM. Differences were considered significant at $95 \%$ confidence level $(* * * P<0.0 I=$ extremely significant; $* * P<0.05=$ very significant, $* P<0 . I=$ significant $),(t$ test). 
For the design of the compartment 2, we have used alginate hydrogel seeded with hybrid MTs (hMSCs and Chondrocytes: $\mathrm{hCHs}$ ). Autologous chondrocytes are used in conventional cell-based therapy, but these techniques are associated with several limitations such as the number of cells available and dedifferentiation in 2D culture. ${ }^{8,42,43}$ By associating hCHs and hMSCs in MTs without the need of chondrogenic medium, we were able to produce chondrogenic MTs, and to prevent their hypertrophy (Figure 2). We assume that by associating hybrid MTs, alginate/HA hydrogel and a membrane equipped with BMP-7 nanoreservoirs, as a new advanced therapeutic medicinal device, we could regenerate first a robust subchondral bone, itself sustaining stable articular cartilage regeneration on its surface.
To demonstrate the behavior of these hybrid MTs in vivo on the membrane $(F)$, we subcutaneously implanted our nanostructured therapeutic medical device (Figure 3A \& 3B). After 90 days, implants were retrieved and cartilage specific staining (GAGs) of the samples was performed (Figure 3C). The two compartments of the implant were visible after 90 days in vivo, showing the bone part of the implant in green (Safranin $\mathrm{O} /$ Fast green) (compartment 1) and the GAGs functional compartment visible in blue (Alcian Blue) or in red (Safranin O/ Fast green) (compartment 2) (Figure 3C). During implantation time, these two compartments were vascularized and cells coming from the microtissues migrated all along the alginate structure and membrane, showing viability of these associated structures (Figure 3C).
A
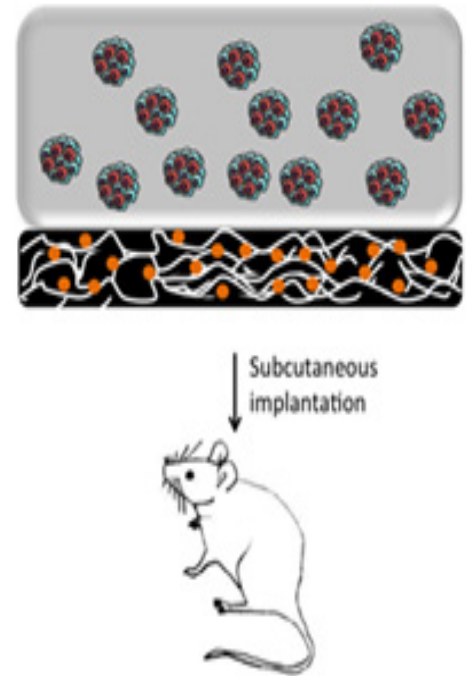

B
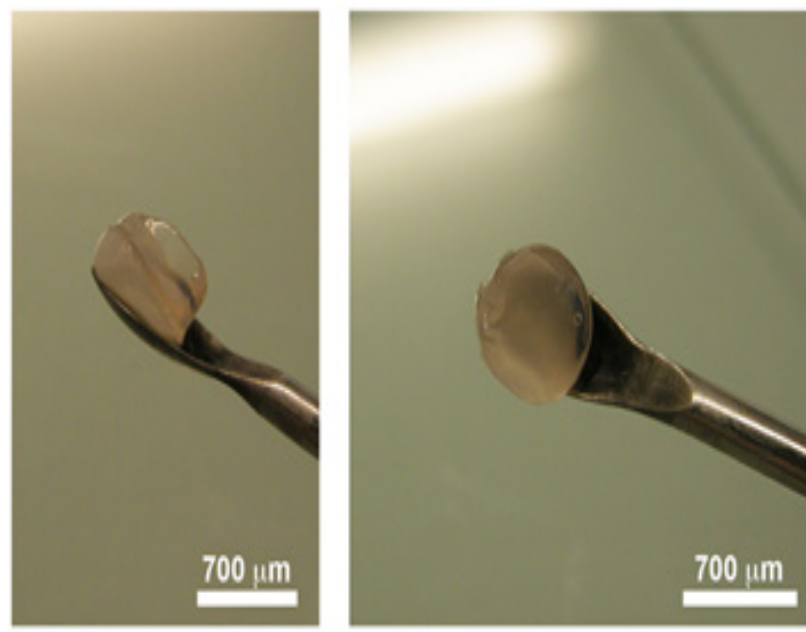

C
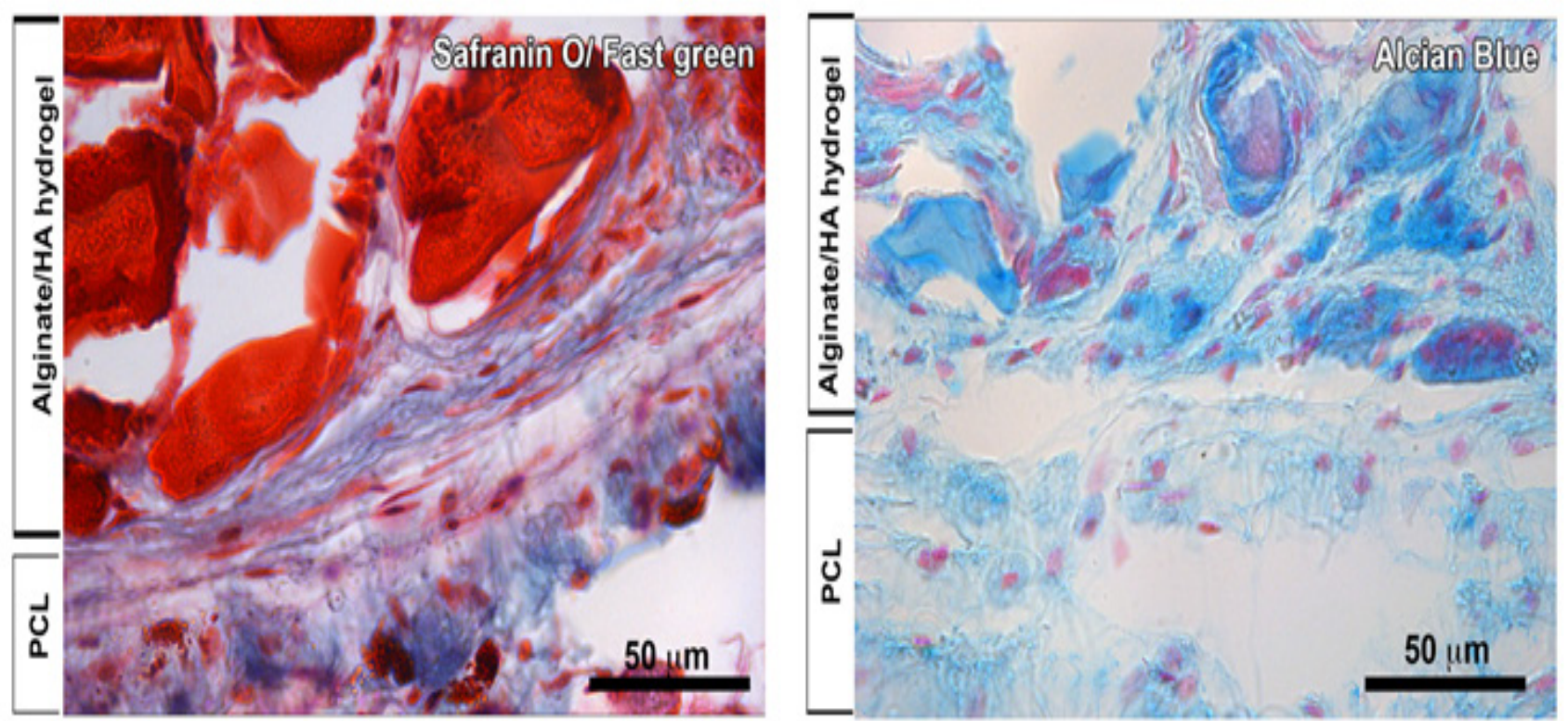

Figure 3 Nanostructured bioactive implant design (compartment I and compartment 2 association) for tuned cartilage hypertrophy. (A,B) The nanofibrous PCL membrane equipped with BMP-7 nanoreservoirs (compartment I) was associated with the alginate/HA hydrogel containing hybrid MTs (compartment 2) and the system was implanted subcutaneously during 90days in nude mice. (C) Safranin O/Fast green and Alcian Blue histological staining showing glycosaminoglycans in red and blue (respectively) in the Alginate/HA compartment compared to the (CHI-BMP-7) ${ }_{6}$ PCL part of the implant. 


\section{Conclusion}

In this manuscript, we used not only a nano fibrous electrospun membrane fortified with active nano reservoirs of therapeutics (BMP7) for subchondral bone repair (compartment 1) but also an appropriate 3D environment for mesenchymal stem cells based on an hydrogel and microtissues for cartilage regeneration with less hypertrophy. These hMSCs were co-cultured with human chondrocytes (hCHs) as inductors in microtissues and placed in some alginate/HA hydrogel (compartment 2). Based on RUNX2 expression, we show that with this double 3D strategy, we are able to modulate the hypertrophic phenotype of the differentiated cells. We have as well-shown in vivo subchondral bone and cartilage regeneration by combining both compartment 1 and 2 .

Success of osteochondral defect treatment can be achieved by targeting not only cartilage repair but also robust subchondral bone. By associating human mesenchymal stem cells/bioactive nano structured material, with alginate hydrogel/3D well-organized cocultured hMSCs, we are able to engineer an implant designed for the articular cartilage but also for the subchondral bone tissue repair. By using a nano fibrous bioactive PCL membrane, and alginate hydrogel combined with stem cells and chondrocytes microtissues, we enhance the cell-cell interactions and optimize the micro environment for subsequent cartilage-bone regeneration. Furthermore, with the use of hybrid microtissues (hCHs, hMSCs) in double 3D environment (alginate /HA, MT), we were able to modulate the hypertrophy of the cells in the articular cartilage compartment. These results demonstrate a promising approach to develop a nano structured and bioactive implantable medical device in the field osteoarticular regenerative nano medicine. Our proposed advanced therapeutic medicinal device opens a new strategy for cartilage repair combining the double 3D active environment of stem cells (Hybrid microtissues in hydrogel) and the nanofibrous active membrane for subchondral bone repair. Our innovative technology based on cells microtissues and hydrogels, as a double $3 \mathrm{D}$ environment, is an adaptable advanced therapeutic medical device. This technology can be tuned by using different kinds of cells, active drugs and polymers (natural or synthetic) and thereby applied for the regeneration of other tissues such as skin and vasculature.

\section{Acknowledgements}

This work was supported by the project Nano OSCAR from the "Agence Nationale de la Recherche", ANR and SATT Conectus. We are indebted to "Faculté de Chirurgie Dentaire" Strasbourg for financial support.

\section{Conflict of interest}

The authors have no other relevant affiliations or financial involvement with any organization or entity with a financial interest in or financial conflict with the subject matter or materials discussed in the manuscript apart from those disclosed.

\section{References}

1. Schwinte P, Keller L, Eap S, et al. Osteoarticular Regenerative Nanomedicine: Advances and Drawbacks in Articular Cartilage Regeneration Implants. Austin J Nanomed Nanotechnol. 2014;2(4):1025.

2. Poole AR, Kojima T, Yasuda T, et al. Composition and structure of articular cartilage:a template for tissue repair. Clin Orthop Relat Res. 2001;(391suppl):S26-S33.
3. Sophia Fox AJ, Bedi A, Rodeo SA. The basic science of articular cartilage:structure, composition, and function. Sports Health. 2009;1(6):461468.

4. Falah M, Nierenberg G, Soudry M, et al. Treatment of articular cartilage lesions of the knee. Int Orthop. 2010;34(5):621-630.

5. Panseri S, Russo A, Cunha C, et al. Osteochondral tissue engineering approaches for articular cartilage and subchondral bone regeneration. Knee Surg Sports TraumatolArthrosc. 2012;20(6):1182-1191.

6. Redman SN, Oldfield SF, Archer CW. Current strategies for articular cartilage repair. Eur Cell Mater. 2005;9:23-32.

7. Matsiko A, Levingstone TJ, OBrien FJ. Advanced Strategies for Articular Cartilage Defect Repair. Materials. 2013;6(2):637-668.

8. Caldwell KL, Wang J. Cell-based articular cartilage repair:the link between development and regeneration. Osteoarthritis Cartilage. 2015;23(3):351-362.

9. Schwintee P, Keller L, Lemoine S, et al. Nano-engineered scaffold for osteoarticular regenerative medicine. J Nanomed Nanotechnol. $2015 ; 6(1): 258$.

10. Frenkel SR, Cesare PED. Scaffolds for articular cartilage repair. Ann Biomed Eng. 2004;32(1):26-34.

11. Spiller KL, Maher SA, Lowman AM. Hydrogels for the repair of articular cartilage defects. Tissue Eng Part B Rev. 2011;17(4):281-299.

12. Madurantakam PA, Cost CP, Simpson DG, et al. Science of nanofibrous scaffold fabrication:strategies for next generation tissue-engineering scaffolds. Nanomedicine (Lond). 2009;4(2):193-206.

13. Zhang YZ, Su B, Venugopal J, et al. Biomimetic and bioactive nanofibrous scaffolds from electrospun composite nanofibers. Int J Nanomedicine. 2007;2(4):623-638.

14. Mendoza-Palomares C, Ferrand A, Facca S, et al. Smart hybrid materials equipped by nanoreservoirs of therapeutics. ACS Nano. 2012;6(1):483490.

15. Ferrand A, Eap S, Richert L, et al. Osteogenetic properties of electrospun nanofibrous PCL scaffolds equipped with chitosan-based nanoreservoirs of growth factors. Macromol Biosci. 2013;14(1):45-55.

16. Rossi F, van Griensven M. Polymer functionalization as a powerfultool to improve scaffold performances. Tissue Eng Part A. 2014;20(1516):2043-2051.

17. Anselme K, Davidson P, Popa AM, et al. The interaction of cells and bacteria with surfaces structured at the nanometre scale. Acta Biomater. 2010;6(10):3824-3846.

18. Wheeldon I, Farhadi A, Bick AG, et al. Nanoscale tissue engineering: spatial control over cell-materials interactions. Nanotechnology. 2011;22(21):212001.

19. Choi JH, Park YW, Park TH, et al. Fuzzy nanoassembly of polyelectrolyte and layered clay multicomposite toward a reliable gas barrier. Langmuir. 2012;28(17):6826-6831.

20. Wang D, Rogach AL, Caruso F. Semiconductor quantum dot-labeled microsphere bioconjugates prepared by stepwise self-assembly. Nano Lett. 2002;2(8):857-861.

21. Berth G, Voigt A, Dautzenberg H, et al. Polyelectrolyte complexes and layer-by-layer capsules from chitosan/chitosan sulfate. Biomacromolecules. 2002;3(3):579-590.

22. Jessel N, Atalar F, Lavalle P, et al. Bioactive coatingsbased on a polyelectrolyte multilayer architecture functionalized by embedded proteins. Advanced Materials. 2003;15(9):692-695. 
23. Hiller J, Mendelsohn JD, Rubner MF. Reversibly erasable nanoporous anti-reflection coatings from polyelectrolyte multilayers. Nature Materials. 2002;1(1):59-63.

24. Benkirane-Jessel N, Schwinte P, Falvey P, et al. Build-up of polypeptide multilayer coatings with anti-inflammatory properties based on the embedding of piroxicam-cyclodextrin complexes. Advanced Functional Materials. 2004;14(2):174-182.

25. Jessel NB, Schwinte P, Donohue R, et al. Pyridylamino- $\beta$-cyclodextrin as a molecular chaperone for lipo polysaccharide embedded in a multilayered polyelectrolyte architecture. Adv Funct Mater. 2004;14(10):963969.

26. Benkirane-Jessel N, Lavalle P, Hübsch E, et al. Short-time tuning of the biological activity of functionalized poly electrolyte multilayers. $A d v$ Funct Mater. 2005;15(4):648-654.

27. Gangloff SC, Ladam G, Dupray V, et al. Biologically active lipid A antagonist embedded in a multilayered polyelectrolyte architecture. Biomaterials. 2006;27(9):1771-1777.

28. Jessel N, Oulad-Abdelghani M, Meyer F, et al. Multiple and timescheduled in situ DNA dnadelivery mediated by beta-cyclodextrin embedded in a polyelectrolyte multilayer. Proc Natl Acad Sci U S A. 2006;103(23):8618-8621.

29. Dierich A, Le Guen E, Messaddeq N, et al. Bone formation mediated by synergy-acting growth factor sembedded in a poly electrolyte multilayer film. Advanced Materials. 2007;19(5):693-697.

30. Zhang X, Sharma KK, Boeglin M, et al. Transfection ability and intracellular DNA pathway of nanostructured gene-delivery systems. Nano Lett. 2008;8(8):2432-2436.

31. Lee J, Kotov NA. Notch ligand presenting acellular $3 \mathrm{~d}$ microenvironments for ex vivo human hematopoietic stem-cell culture made by layerby-layer assembly. Small. 2009;5(9):1008-1013.

32. Shim BS, Podsiadlo P, Lilly DG, et al. Nanostructured thin films made by dewetting method of layer-by-layer assembly. Nano Lett. 2007;7(11):3266-3273.

33. Eap S, Ferrand A, Schiavi J, et al. Collagen implants equipped with "fish scale"-like nanoreservoirs of growth factors for bone regeneration. Nanomedicine (Lond). 2014;9(8):1253-1261.

34. Facca S, Cortez C, Mendoza-Palomares C, et al. Active multilayered capsules for in vivo bone formation. Proc Natl Acad Sci USA. 2010;107(8):3406-3411.

35. Schiavi J, Keller L, Morand DN, et al. Active implant combining human stem cell microtissues and growth factors for bone-regenerative nanomedicine. Nanomedicine (Lond). 2015;10(5):753-763.

36. Eap S, Keller L, Schiavi J, et al. A living thick nanofibrous implant bifunctionalized with active growth factor and stem cells for bone regeneration. Int J Nanomedicine. 2015;10:1061-1075.

37. Decher G. Fuzzy nanoassemblies: toward layered polymericmulti composites. Science. 1997;277(5330):1232-1237.

38. Magne D, Vinatier C, Julien M, et al. Mesenchymal stem cell therapy to rebuild cartilage. Trends Mol Med. 2005;11(11):519-526.

39. Woods A, Wang G, Beier F. Regulation of chondrocyte differentiation by the actin cytoskeleton and adhesive interactions. $J$ Cell Physiol. 2007;213(1):1-8.

40. Beane OS, Darling EM. Isolation, characterization, and differentiation of stem cells for cartilage regeneration. Ann Biomed Eng. 2012;40(10):2079-2097.

41. Seo S, Na K. Mesenchymal stem cell-based tissue engineering for chondrogenesis. J Biomed Biotechnol. 2011;2011:80689-806891.
42. Von der Mark K, Gauss V, von der Mark H, et al. Relationship between cell shape and type of collagen synthesised as chondrocytes lose their cartilage phenotype in culture. Nature. 1977;267(5611):531-532.

43. Benya PD, Padilla SR, Nimni ME. Independent regulation of collagen types by chondrocytes during the loss of differentiated function in culture. Cell. 1978;15(4):1313-1321.

44. Wolf F, Candrian C, Wendt D, et al. Cartilage tissue engineering using pre-aggregated human articular chondrocytes. Eur Cell Mater. 2008;16:92-99.

45. Lehmann M, Martin F, Mannigel K, et al. Three-dimensional scaffoldfree fusion culture:the way to enhance chondrogenesis of in vitro propagated human articular chondrocytes. Eur J Histochem. 2013;57(4):e31.

46. Frenz DA, Jaikaria NS, Newman SA. The mechanism of precartilage mesenchymal condensation:a major role for interaction of the cell surface with the amino-terminal heparin-binding domain of fibronectin. Dev Biol. 1989;136(1):97-103.

47. DeLise AM, Fischer L, Tuan RS. Cellular interactions and signaling in cartilage development. Osteoarthritis Cartilage. 2000;8(5):309-334.

48. Singh P, Schwarzbauer JE. Fibronectin and stem cell differentiation lessons from chondrogenesis. J Cell Sci. 2012;125(16):3703-3712.

49. Fennema E, Rivron N, Rouwkema J, et al. Spheroid culture as a tool for creating 3d complex tissues. Trends in Biotechnol. 2013;31(2):108-115.

50. Nooeaid P, Salih V, Beier JP, et al. Osteochondral tissue engineering: scaffolds, stem cells and applications. J Cell Mol Med. 2012;16(10):22472270 .

51. Nukavarapu SP, Dorcemus DL. Osteochondral tissue engineering: current strategies and challenges. Biotechnol Adv. 2013;31(5):706-721.

52. Chen J, Chen H, Li P, et al. Simultaneous regeneration of articular cartilage and subchondral bone in vivo using MSCS induced by a spatially controlled gene delivery system in bilayered integrated scaffolds. Biomaterials. 2011;32(21):4793-4805

53. Yan LP, Silva-Correia J, Oliveira MB, et al. Bilayered silk/silk-nano Cap scaffolds for osteochondral tissue engineering: in vitro and in vivo assessment of biological performance. Acta Biomater. 2015;12:227-241.

54. Liu M, Yu X, Huang F, et al. Tissue engineering stratified scaffolds for articular cartilage and subchondral bonedefects repair. Orthopedics. 2013;36(11):868-873

55. Steele JA, McCullen SD, Callanan A, et al. Combinatorial scaffold morphologies for zonal articular cartilage engineering. Acta Biomater. 2014;10(5):2065-2075.

56. Porter JR, Ruckh TT, Popat KC. Bone tissue engineering: a review in bone biomimetics and drug delivery strategies. Biotechnol Prog. 2009;25(6):1539-1560.

57. Lee SH, Shin H. Matrices and scaffolds for delivery of bioactive molecules in bone and cartilage tissue engineering. Adv Drug Deliv Rev. 2007;59(4-5):339-359.

58. Kanakaris NK, Mallina R, Calori GM, et al. Use of bone morphogenetic proteins in arthrodesis:clinical results. Injury. 2009;40(Suppl 3):S62S66.

59. Govender S, Csimma C, Genant HK, et al. Recombinant human bone morphogenetic protein-2 for treatment of open tibial fractures: a prospective, controlled, randomized study of four hundred and fifty patients. J Bone Joint Surg Am. 2002;84-A(12):2123-2134.

60. Lavery K, Hawley S, Swain P, et al. New insights into BMP-7 mediated osteoblastic differentiation of primary human mesenchymal stem cells Bone. 2009;45(1):27-41. 
61. Cooke ME, Allon AA, Cheng T, et al. Structured three-dimensional coculture of mesenchymal stem cells with chondrocytes promotes chondrogenic differentiation without hypertrophy. Osteoarthritis Cartilage. 2011;19(10):1210-1218

62. Hubka KM, Dahlin RL, Meretoja VV, et al. Enhancing chondrogenic phenotype for cartilage tissue engineering: monoculture and coculture of articular chondrocytes and mesenchymal stem cells. Tissue Eng Part B Rev. 2014;20(6):641-654.

63. Meretoja VV, Dahlin RL, Kasper FK, et al. Enhanced chondrogenesis in co-cultures with articular chondrocytes and mesenchymal stem cells. Biomaterials. 2012;33(27):6362-6369.

64. Tsuchiya K, Chen G, Ushida T, et al. The effect of coculture of chondrocytes with mesenchymal stem cells on theircartil aginous phenotype in vitro. Materials Science and Engineering: C. 2004;24(3):391-396.

65. Caron MM, Emans PJ, Coolsen MM, et al. Redifferentiation of dedifferentiated human articular chondrocytes:comparison of 2D and 3D cultures. Osteoarthr Cartilage. 2012;20(10):1170-1178.
66. Holtzer H, Abbott J, Lash J, et al. The loss of phenotypic traits by differentiated cells in vitro, I. dedifferentiation of cartilage cells. Proc Natl Acad Sci U S A. 1960;46(12):1533-1542.

67. Lai JH, Kajiyama G, Smith RL, et al. Stem cells catalyze cartilage formation by neonatal articular chondrocytes in $3 \mathrm{~d}$ biomimetic hydrogels. Sci Rep. 2013;3(3553):1-9.

68. Lim F, Sun AM. Microencapsulated islets as bioartificial endocrine pancreas. Science. 1980;210(4472):908-910.

69. Atala A, Kim W, Paige KT, et al. Endoscopic treatment of vesicoureteral reflux with a chondrocyte-alginate suspension. J Urol. 1994;152(2 Pt 2):641-643.

70. Pokrywczynska M, Drewa T, Jundzill A, et al. Alginate is not a good material for growth of rapidly proliferating cells. Transplant Proc. 2008;40(5):1664-1667.

71. Rowley JA, Madlambayan G, Mooney DJ. Alginate hydrogels as synthetic extracellular matrix materials. Biomaterials. 1999;20(1):45-53. 